\title{
From Homer and Hippocrates to modern personalized medicine: is there a role for pharmacoepigenomics in the treatment of alcohol addiction?
}

\author{
Georgia Ragia*,1,2 \& Vangelis G Manolopoulos ${ }^{1,3}$ \\ ${ }^{1}$ Laboratory of Pharmacology, Medical School, Democritus University of Thrace, Dragana Campus, 68100 Alexandroupolis, Greece \\ ${ }^{2}$ DNALEX S.A., Leontaridou 2, Alexandroupolis, Greece \\ ${ }^{3}$ Pharmacology \& Pharmacogenetics Unit, Academic General Hospital of Evros, Alexandroupolis, Greece \\ * Author for correspondence: Tel...+30 255103 0523; Fax: +30 255103 0523; gragia@med.duth.gr
}

"For sensible men I prepare only three kraters: one for health to drink first, the second for love and pleasure and the third for sleep. After the third one is drained, wise men go home. The fourth krater is not mine anymore - it belongs to bad behavior"

From the earliest times to the present, alcohol has evolved as part of life and culture. For most adults, moderate alcohol use is harmless, however, it lies at one end of a range that moves through alcohol abuse to alcohol addiction. Alcohol addiction is a serious and chronic psychiatric disorder that, on top of its heavy consequences on health, also brings significant social and economic losses to individuals and society at large. Pharmacotherapy of alcohol addiction exists, but its effectiveness varies significantly among individuals. Genomic and nongenomic factors are significant contributors to interindividual variation in the clinical presentation of alcohol problems and the response to a given treatment. In addition, emerging evidence suggests pharmacoepigenomics of alcohol addiction as a novel promising area for improvement of alcohol addiction management.

First draft submitted: 19 December 2017; Accepted for publication: 23 February 2018; Published online: 27 March 2018

Keywords: addiction $\bullet$ alcohol $\bullet$ Dionysus $\bullet$ pharmacoepigenomics $\bullet$ pharmacogenomics

\section{From Dionysus festivals to alcohol addiction}

From the earliest times to the present, alcohol has played an important role in religion and worship. Late stone beer jugs suggest that alcoholic beverages such as primitive beers were used since the dawn of civilization, while wine has evolved as part of life, culture and diet since time immemorial. Wine making reached the Hellenic peninsula at about $2000 \mathrm{BC}$ and helped Ancient Greece trade with neighboring countries and regions [1]. Drinking was central to Greek culture, as evidenced by special drinking-party-poetry to the festivals of Bacchus, also known as Dionysus, the god of wine. The two great epic poems of Homer, Iliad and Odyssey, are suffused with references to wine and its powers [2,3], while wine was the central spirit of Greek symposia. Hippocrates, the father of western medicine, advocated the use of wine to treat every illness he had identified [4] with the exception of patients suffering from 'an overpowering heaviness of the brain', in whom 'there must be total abstinence from wine'. Ancient Greeks, however, early on identified the dangers of excessive drinking that may even lead to early death. Wine was a gift from the gods but not to be over-indulged in or one would end up meeting them earlier than one hoped. Additionally, from Homeric time alcohol intoxication provoked social contempt and heavy drinkers were considered as reprehensible and punishable for mimicking the barbarians. Nowadays, drinking alcohol is a common feature of social gatherings. For most adults, moderate alcohol use is relatively harmless, however, it lies at one end of a range that moves through alcohol abuse to alcohol addiction. 


\section{The burden of alcohol addiction}

Alcohol addiction, also called alcohol dependence or alcoholism, is a serious and chronic psychiatric disorder. It harbors a wide range of harmful physical, mental and social consequences. Systematic consumption of large amounts of alcohol is linked to the development of chronic diseases and is associated with an increased risk of acute health conditions, such as injuries, including those from traffic accidents. According to the WHO fact sheet for alcohol, worldwide, 3.3 million deaths every year (5.9\% of all global deaths) result from harmful use of alcohol. The harmful use of alcohol is a causal factor in more than 200 disease and injury conditions $(5.1 \%$ of the global burden of disease and injury). Beyond health consequences, the harmful use of alcohol brings significant social and economic losses to individuals and society at large [5].

\section{Alcohol addiction treatment}

Treatment methods for alcoholism combine medications, behavioral treatment and peer support to retain abstinence and prevent long-term relapse. A pharmacological adjunct to psychosocial therapy is necessary in most of the cases, since without pharmacotherapy the clinical outcome is poor, with up to $70 \%$ of patients resuming drinking within 1 year $[6,7]$.

Currently, disulfiram, naltrexone and acamprosate are the drugs approved by the US FDA for treatment of alcohol dependence. Nalmefene has been approved in Europe in 2013, but is not yet approved in the USA for alcohol addiction. Options for alcohol addiction pharmacotherapy are limited and several other compounds have been tested for alcohol dependence treatment, some of them currently being at various stages of clinical development. Interestingly, a series of compounds, clinically approved for indications other than alcohol addiction, appear to have the ability to reduce alcohol consumption [6,7]. The most recent example is an anti-inflammatory drug, ibudilast, primarily used in asthma, that appears to reduce cravings and stress-related symptoms [8]. Additionally, the pharmacologic treatment of alcohol addiction includes several other drugs used in psychiatry, such as anxiolytics and antidepressants, that are used to treat withdrawal symptoms and to prevent seizures and delirium [9].

\section{Interindividual variability in response to alcohol addiction pharmacotherapy: nongenomic \& genomic factors}

Effectiveness of pharmacotherapy of alcohol addiction varies among individuals. Evidence from a number of different studies suggests that different factors are significant contributors to interindividual variation both in the clinical presentation of alcohol problems as well as in the response to a given treatment [10]. Multiple biological and psychosocial factors mutually influence each other in causing alcohol abuse and in interfering with response to pharmacotherapy. Motivation, psychological support and socioeconomic status are among the most important nongenomic factors associated with both alcohol addiction and alcohol addiction therapy response and are vital in order to prevent relapse [6,7].

Genomic factors are also important determinants of both alcohol addiction and response to pharmacotherapy. Gene variations and gene-gene interactions govern the high heritability rate of alcohol addiction and have a dominant role in mediating alcohol reward properties and the phenotypic characteristics of the neurobiological mechanism of alcohol addiction. Among the genes that are associated with alcohol addiction are from components of the serotonergic, dopaminergic, opioid and glutaminergic systems as well as genes encoding the alcoholmetabolizing enzymes [11,12]. In terms of responding to pharmacotherapy, pharmacogenomics is paving the path to personalized medicine of alcohol addiction [6,7]. For all three FDA-approved anticraving drugs, gene variants have been assessed for their effect on response to pharmacotherapy, however, naltrexone is by far the most well studied drug and its pharmacogenomic application is mature for clinical application. OPRM1 A118G polymorphism has emerged as a potentially useful genomic marker in personalizing naltrexone treatment with the OPRM1 118G allele being a predictor of good naltrexone treatment response. For disulfiram, $D \beta H \mathrm{C}-1021 \mathrm{~T}$ polymorphism, that affects circulating $\mathrm{D} \beta \mathrm{H}$ levels, is the only gene that has been assessed in relation to disulfiram response in alcohol addicted individuals, whereas for acamprosate seminal data indicate the potential association of $G A B R A G$, GABRB2, DRD2 and GRIN2B gene polymorphisms with response [6,7].

\section{Epigenomics \& pharmacoepigenomics of alcohol addiction}

Epigenomics is the field that focuses on nongenomic modifications that influence gene expression, such as DNA methylation and histone acetylation. Additionally, though not directly associated with gene expression, miRNAs are often considered part of epigenomics as potent regulators of gene translation [13]. 
Ethanol exposure leads to gene expression changes, therefore in alcohol addiction, several epigenomic modifications exist. Changes in gene expression in brain reward regions are thought to contribute to the pathogenesis and persistence of alcohol addiction. Neuroadaptations observed in alcoholism are attributed to changes in gene expression due to histone modifications and DNA methylation. Additionally, the well-known effects of alcohol on lipid metabolism and cardiovascular risk may also be partially mediated via epigenomic modifications [14]. Postmortem studies in brains of alcohol addicted patients indeed showed significant differences in the methylation pattern of more than 400 CpGs between patients and controls [15] and several of these alterations are located in the prefrontal cortex [16]. Additionally, in alcohol addiction, miRNA regulation of different proteins following alcohol exposure may lead to alterations in neurotransmission [17].

Epigenomics expands the scope of pharmacogenomics toward optimization of drug therapy, leading to the introduction of a new term, namely pharmacoepigenomics, that reflects the combined analysis of genomic variations and epigenomic modifications [13]. Pharmacoepigenomics of alcohol addiction has just started to emerge. Drugs that, directly or indirectly, act on epigenomic mechanisms, appear to modify drinking habits and alcohol effects. A recent study in rats has shown that valproic acid, a HDAC inhibitor, reduces the preference for, and the consumption of, ethanol [18]. More recently, in a chronic alcohol exposure rat animal model, 5-Aza-2'-deoxycytidine, an inhibitor of DNA methyltransferases approved for myelodysplastic syndrome treatment, decreased alcohol consumption and alcohol preference [19]. Additionally, in a mouse paradigm that mimics binge alcohol drinking in humans, it was shown that inhibition of miR-30a-5p restored BDNF levels and decreased excessive alcohol intake [20]. These few studies consist promising preliminary evidence that pharmacoepigenomics of alcohol addiction may be a significant field of research. The deeper understanding of the connection between alcohol consumption, epigenomic modifications and illness and disease could help researchers in tailoring existing, and/or developing novel, medications or therapies for alcohol addiction prevention or treatment.

\section{From Hippocrates to modern personalized medicine}

Personalized medicine cannot be considered a modern term. Hippocrates who is traditionally regarded as the father of western medicine wrote about the individuality of disease and the necessity of giving "different drugs to different patients, for the sweet ones do not benefit everyone, nor do the astringent ones, nor are all the patients able to drink the same things". Could he be regarded also as the father of personalized medicine? Hippocrates evaluated factors such as a person's 'constitution', age and 'physique', as well as the time of year, to aid his decision making when prescribing drugs [21]. Several centuries later, 21st century personalized medicine has evolved and incorporates current knowledge on different 'omics', including genomics, pharmacogenomics, epigenomics, pharmacoepigenomics and metabolomics.

\section{Is there a role for pharmacoepigenomics in the treatment of alcohol addiction?}

Presently, DNA methylation and histone acetylation are by far the most substantially studied epigenomic mechanisms. Data indicate that drugs modifying epigenomic alterations hold promise to advocate the management of alcohol addiction. Currently, however, no drug targeting epigenomic mechanisms is approved for alcohol or other substance addiction. It should also be recognized that a main limitation of alcohol addiction pharmacoepigenomics is that epigenomics in general is tissue specific and there is the need of identifying similar methylation patterns in global markers. Further research in the field warrants the identification of genes that are epigenetically modified and can serve either as target for novel therapeutic compounds or as markers of patients response to a given pharmacotherapy. It is anticipated that in the near future pharmacoepigenomics of alcohol addiction will become a tangible tool of dependence personalized medicine.

In all cases, drinkers should keep in mind the words by the god of wine himself: "For sensible men I prepare only three kraters: one for health to drink first, the second for love and pleasure and the third for sleep. After the third one is drained, wise men go home. The fourth krater is not mine anymore - it belongs to bad behavior; the fifth is for shouting; the sixth is for rudeness and insults; the seventh is for fights; the eighth is for breaking the furniture; the ninth is for depression; the tenth is for madness and unconsciousness.?

Financial \& competing interests disclosure

The authors have no relevant affiliations or financial involvement with any organization or entity with a financial interest in or financial conflict with the subject matter or materials discussed in the manuscript. This includes employment, consultancies, honoraria, stock ownership or options, expert testimony, grants or patents received or pending, or royalties.

No writing assistance was utilized in the production of this manuscript. 


\section{References}

1. Younger WA. Gods, Men, and Wine. Michael Joseph, London, UK (1966).

2. Fagles R. Homer-The Iliad. Penguin Press, NY, USA (1990).

3. Fagles R. Homer - The Odyssey. Penguin Press, NY, USA (1996).

4. Hippocrates, Galen, Coxe JR. The Writings of Hippocrates and Galen. Linday and Blakiston, PA, USA (1846).

5. WHO. Global status report on alcohol and health (2014). http://apps.who.int/iris/bitstream/10665/112736/1/9789240692763_eng.pdf?ua=1

6. Ragia G, Manolopoulos VG. Personalized medicine of alcohol addiction: pharmacogenomics and beyond. Curr. Pharm. Biotechnol. 18(3), 221-230 (2017).

7. Ragia G, Manolopoulos VG. Pharmacogenomics of alcohol addiction: personalizing pharmacologic treatment of alcohol dependence. Hosp. Pharmacol. 1(13), 147-167 (2014).

8. Ray LA, Bujarski S, Shoptaw S, Roche DJ, Heinzerling K, Miotto K. Development of the neuroimmune modulator ibudilast for the treatment of alcoholism: a randomized, placebo-controlled, human laboratory trial. Neuropsychopharmacology 42(9), 1776-1788 (2017).

9. Michalak A, Biala G. Alcohol dependence - neurobiology and treatment. Acta Pol. Pharm. 73(1), 3-12 (2016).

10. Ferraguti G, Pascale E, Lucarelli M. Alcohol addiction: a molecular biology perspective. Curr. Med. Chem. 22(6), 670-684 (2015).

11. Ragia G, Iordanidou M, Giannakopoulou E, Tavridou A, Manolopoulos VG. Association of DRD2 TaqIA and D $\beta H-1021 C>T$ gene polymorphisms with smoking initiation and their interaction with serotonergic system gene polymorphisms. 11(2), 110-117 (2013).

12. Ragia G, Veresies I, Veresie L, Veresies K, Manolopoulos VG. Association study of DRD2 A2/A1, DRD3 Ser9Gly, DbetaH -1021C>T, OPRM1 A118G and GRIK1 rs2832407C>A polymorphisms with alcohol dependence. Drug Metab. Pers. Ther. 31(3), 143-150 (2016).

13. Manolopoulos VG, Ragia G, Tavridou A. Pharmacogenomics of oral antidiabetic medications: current data and pharmacoepigenomic perspective. Pharmacogenomics 12(8), 1161-1191 (2011).

14. Lohoff FW, Sorcher JL, Rosen AD et al. Methylomic profiling and replication implicates deregulation of PCSK9 in alcohol use disorder. Mol. Psychiatry doi:10.1038/mp.2017.168 (2017) (Epub ahead of print).

15. Hagerty SL, Bidwell LC, Harlaar N, Hutchison KE. An exploratory association study of alcohol use disorder and DNA methylation. Alcohol Clin. Exp. Res. 40(8), 1633-1640 (2016).

16. Wang F, Xu H, Zhao H, Gelernter J, Zhang H. DNA co-methylation modules in postmortem prefrontal cortex tissues of European Australians with alcohol use disorders. Sci. Rep. 6, 19430 (2016).

17. Mathew DE, Larsen K, Janeczek P, Lewohl JM. Expression of 14-3-3 transcript isoforms in response to ethanol exposure and their regulation by miRNAs. Mol. Cell Neurosci. 75, 44-49 (2016).

18. Al Ameri M, Al Mansouri S, Al Maamari A, Bahi A. The histone deacetylase (HDAC) inhibitor valproic acid reduces ethanol consumption and ethanol-conditioned place preference in rats. Brain Res. 1583, 122-131 (2014).

19. Qiao X, Yin F, Ji Y, Li Y, Yan P, Lai J. 5-Aza-2'-deoxycytidine in the medial prefrontal cortex regulates alcohol-related behavior and Ntf3-TrkC expression in rats. PLoS ONE 12(6), e0179469 (2017).

20. Darcq E, Warnault V, Phamluong K, Besserer GM, Liu F, Ron D. microRNA-30a-5p in the prefrontal cortex controls the transition from moderate to excessive alcohol consumption. Mol. Psychiatry 20(10), 1261 (2015).

21. Kleisiaris CF, Sfakianakis C, Papathanasiou IV. Healthcare practices in ancient Greece: the Hippocratic ideal. J. Med. Ethics Hist. Med. 7 , 6 (2014). 